\title{
On Conditionally Convergent Series
}

\author{
Werner Horn and Madjiguene Ndiaye ${ }^{*}$
}

\begin{abstract}
We prove conditions of convergence for rearrangements of conditionally convergent series. The main results are a comparison theorem using integrals and a limit comparison theorem for rearrangements. This is done by using elementary techniques from calculus.
\end{abstract}

\section{INTRODUCTION}

In most calculus courses students learn that the terms of a conditionally convergent series

$$
T=\sum_{k=1}^{\infty} a_{k}(-1)^{k-1}
$$

may be rearranged to converge to any given real number. This result is somewhat mysterious as it seems to contradict our experience. After all the commutativity of addition of real numbers is one of the truth', which we hold as self-evident. Why should this principle break down, when the sum contains an infinite number of terms. Surprisingly, the proof of this result is relatively easy and we will start with a rough sketch of it before shedding some different light on this result. If the sequence $\left\{a_{k}\right\}_{k=1}^{\infty}$ is a decreasing (or at least eventually decreasing) positive sequence that

*both authors are at the Department of Mathematics, California State University Northridge, 18111 Nordhoff St., Northridge, CA 91330-8313

†Both authors were partially supported by NSF Grant DMS - 0502258 
converges to 0 , and $A$ is a positive real number, one obtains a rearrangement that converges to $A$ by first adding odd (positive) terms in the series until

$$
\sum_{k=1}^{N-1} a_{2 k-1}<A,
$$

and

$$
\sum_{k=1}^{N} a_{2 k-1} \geq A .
$$

In the next step one subtracts one or more of the negative terms until the sum is less than $A$. One continues this process by adding positive terms until the sum exceeds $A$ and subtracting negative terms until it is less than $A$ again. In this manner $A$ becomes sandwiched between the partial sums ending with a negative term and the ones ending with a positive term. And since $a_{k}$ is decreasing to zero, the difference of these partial sums will also go to zero. We refer the reader to [6, pp. 318-319] or $[4$, p. 518] for a complete proof of this result.

In this paper we will investigate this result from a slightly different angle. For a given rearrangement the $N$-th partial sum

$$
S_{N}(A)
$$

contains a unique number $p_{N}$ of positive terms and $q_{N}$ of negative terms. In this way the rearrangement can be identified with two sequences of integers

$$
\left\{p_{N}\right\}_{N=1}^{\infty}, \quad \text { and } \quad\left\{q_{N}\right\}_{N=1}^{\infty}
$$

We will first prove a result that connects these sequences in a simple formula to the limit of the rearranged series. This will allow us to compute limits of the series in an efficient way and will lead us to criteria for the convergence of rearrangements. These results are not original, and similar results on the relation between the limits of the series and these two sequences (or related sequences) have appeared in the literature before. A rather complete treatment of these and related problems was given by A. Pringsheim as early 1883 [7]. However, this paper is not easily accessible 
to most students, as it was written in German and in a rather archaic style. More recent works on this and related subjects are $[2,3,8]$. Despite the richness of the literature, we felt that the subject deserves further investigation. The proofs of the theorems in this paper are completely elementary and accessible to anyone with a strong background in calculus. In the next section we will state and prove the main result on the relationship between the limits of the series and the sequences $\left\{p_{N}\right\}_{N=1}^{\infty}$ and $\left\{q_{N}\right\}_{N=1}^{\infty}$. The third section of the paper will cover some consequences and simple examples. In the final section we will use the earlier results to prove a limit comparison theorem for rearrangements of series. We will limit ourselves to series with decreasing or eventually decreasing terms.

\section{THE MAIN RESULT}

To state and prove the main result we will first introduce some notation, namely let

$$
\left\{a_{k}\right\}_{k=1}^{\infty}
$$

be a non-negative sequence, that converges to zero and is eventually decreasing.Moreover, let $f$ be a continuous, non-negative, and eventually decreasing function on $[1, \infty)$ such that

$$
f(k)=a_{k},
$$

for all positive integers $k$. Such a function will always exist, since one can just take the piecewise linear function connecting the points $\left(k, a_{k}\right)$. Define

$$
F(x)=\int_{1}^{x} f(t) d t
$$

The alternating series

$$
T=\lim _{N \rightarrow \infty} T_{N}=\lim _{N \rightarrow \infty} \sum_{k=1}^{N} a_{k}(-1)^{k-1}
$$

converges by the alternating series test. If in addition $F$ is bounded the series converges absolutely. 
To continue let

$$
S_{N}(A)
$$

be the $N$-th partial sum of a rearrangement of the series that converges to a real number $A$, and let $\left\{p_{N}\right\}_{N=1}^{\infty}$ and $\left\{q_{N}\right\}_{N=1}^{\infty}$ be the related sequences of positive and negative terms mentioned above. It is easily checked that

$$
p_{N}+q_{N}=N
$$

and

$$
S_{N}(A)=\sum_{k=1}^{p_{N}} a_{2 k-1}-\sum_{k=1}^{q_{N}} a_{2 k} .
$$

We can now state the main result of this note:

Theorem 1 With the notations introduced above we have:

$$
\lim _{N \rightarrow \infty}\left(F\left(2 p_{N}\right)-F\left(2 q_{N}\right)\right)=2 A-2 T
$$

Proof: Without loss of generality, we will assume that $p_{N} \geq q_{N}$ for all sufficiently large values of $N$. The other case can be shown in the completely analogous way. We have

$$
\begin{aligned}
S_{N}(A) & =\sum_{k=1}^{p_{N}} a_{2 k-1}-\sum_{k=1}^{q_{N}} a_{2 k} \\
& =\sum_{k=1}^{2 q_{N}} a_{k}(-1)^{k-1}+\sum_{k=q_{N}+1}^{p_{N}} a_{2 k-1} \\
& =T_{2 q_{N}}+\sum_{k=q_{N}+1}^{p_{N}} a_{2 k-1},
\end{aligned}
$$

where $T_{2 q_{N}}$ is the $2 q_{N}$-th partial sum for the original alternating series. From Figure 1 below we see that

$$
2 \sum_{k=q_{N}+2}^{p_{N}} a_{2 k-1} \leq \int_{2 q_{N}}^{2 p_{N}} f(t) d t
$$

and therefore

$$
2 \sum_{k=q_{N}+1}^{p_{N}} a_{2 k-1}-2 a_{2 q_{N}+1} \leq \int_{2 q_{N}}^{2 p_{N}} f(t) d t .
$$


Figure 2 shows that

$2 \sum_{k=q_{N}}^{p_{N}} a_{2 k-1} \geq \int_{2 q_{N}}^{2 p_{N}} f(t) d t$

and therefore

$2 \sum_{k=q_{N}+1}^{p_{N}} a_{2 k-1}+2 a_{2 q_{N}-1} \leq \int_{2 q_{N}}^{2 p_{N}} f(t) d t$

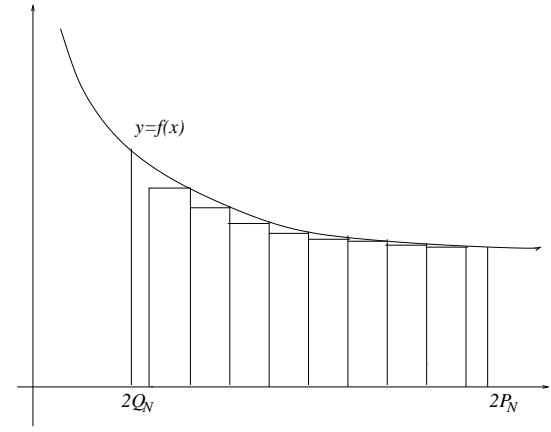

Figure 1: Upper estimate of the sum by the integral

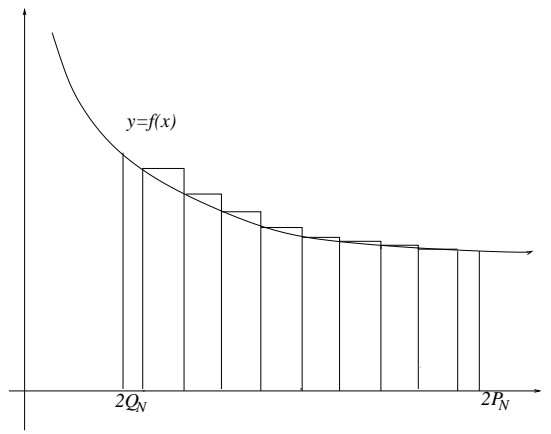

Figure 2: Lower estimate of the sum by the integral

Combining (4) and (5), we obtain

$$
\int_{2 q_{N}}^{2 p_{N}} f(t) d t-2 a_{2 q_{N}-1}-2 a_{2 q_{N}-1} \leq 2 \sum_{k=q_{N}+1}^{p_{N}} a_{2 k-1} \leq \int_{2 q_{N}}^{2 p_{N}} f(t) d t+2 a_{2 q_{N}+1},
$$

which immediately implies

$$
\int_{2 q_{N}}^{2 p_{N}} f(t) d t-2 a_{2 q_{N}-1}-2 a_{2 q_{N}-1} \leq 2 S_{N}(A)-2 T_{2 q_{N}} \leq \int_{2 q_{N}}^{2 p_{N}} f(t) d t+2 a_{2 q_{N}+1} .
$$

Finally, we observe that

$$
\lim _{N \rightarrow \infty} 2 a_{2 q_{N}-1}=\lim _{N \rightarrow \infty} 2 a_{2 q_{N}+1}=0 .
$$

Therefore the desired result follows from taking the limit as $N \rightarrow \infty$ in all terms of (6). 
Corollary 1 If $\lim _{x \rightarrow \infty} F(x)$ exists and is finite, the series is absolutely convergent, and

$$
\lim _{N \rightarrow \infty}\left(F\left(2 p_{N}\right)-F\left(2 q_{N}\right)\right)=0 .
$$

Therefore, any rearrangement of an absolutely convergent series converges to the same limit.

Proof: In this case we have

$$
\lim _{N \rightarrow \infty} F\left(2 p_{N}\right)=\lim _{N \rightarrow \infty} F\left(2 q_{N}\right)
$$

and the result follows immediately.

\section{EXAMPLES AND CONSEQUENCES}

In this section we will use Theorem 1 to investigate the convergence of rearrangements of some prominent alternating series. In all cases we do the explicit proofs for $p_{N} \geq q_{N}$ (at least eventually). We mention that the other situation can be handled in the same way. The most prominent - and most intensively investigated conditionally convergent series is the alternating harmonic series, indeed several of the papers cited specialize on this topic $[3,5]$. As we will later see this series is not a very good model, since it is converging so rapidly, in fact it is almost absolutely convergent. Applying Theorem 1 to that series yields the following result.

Corollary 2 For the alternating harmonic series the statement of Theorem 1 becomes:

$$
A-T=\frac{1}{2} \lim _{N \rightarrow \infty} \ln \frac{p_{N}}{q_{N}}
$$

Proof: In this case $f(t)=\frac{1}{t}$ and $F(t)=\ln t$. Applying Theorem 1 gives

$$
2 A-2 T=\lim _{N \rightarrow \infty}\left(\ln 2 p_{N}-\ln 2 q_{N}\right)=\lim _{N \rightarrow \infty} \frac{p_{N}}{q_{N}} .
$$


In particular, this Corollary implies that the limit of a rearrangement of the alternating harmonic series is finite, if and only if $\lim _{N \rightarrow \infty} \frac{p_{N}}{q_{N}}$ is finite. Other authors do not use the ratio we use in this paper, but rather use the asymptotic density of positive terms in the rearrangement, which is defined as

$$
\rho=\lim _{N \rightarrow \infty} \frac{p_{N}}{N} .
$$

In this notation, the above corollary implies that a rearrangement of the alternating series converges to a finite limit if and only if $0<\rho<1$. The alternating harmonic series is a relatively rapidly converging alternating series and represents as such a limiting case for conditionally convergent series. Corollary 1 also allows us to compute explicit rearrangements converging to a given number. Since in this case it is known that $T=\ln 2$. For example, to construct a rearrangement which converges to $A=\ln 3$ we must ensure that

$$
\frac{1}{2} \lim _{N \rightarrow \infty} \ln \frac{p_{N}}{q_{N}}=\ln 3-\ln 2=\ln \frac{3}{2} .
$$

This can easily be achieved by taking always 9 positive terms followed by 4 negative terms.

In our next example we will investigate a class of slower converging $p$-series.

Corollary 3 Consider the alternating p-series

$$
T=\sum_{k=1}^{\infty} \frac{(-1)^{k-1}}{k^{p}}
$$

with $0<p<1$. Then a rearrangement of this series converges to a finite limit if and only if

$$
\lim _{N \rightarrow \infty} \frac{p_{N}}{q_{N}}=1
$$

i.e. $\rho=\frac{1}{2}$.

Proof: In this case $f(t)=t^{-p}$ and hence

$$
F(t)=\frac{1}{1-p}\left(t^{1-p}-1\right) .
$$


The theorem implies that

$$
2 A-2 T=\lim _{N \rightarrow \infty} \frac{1}{1-p}\left(\left(2 p_{N}\right)^{1-p}-\left(2 q_{N}\right)^{1-p}\right)=\lim _{N \rightarrow \infty} \frac{\left(2 q_{N}\right)^{1-p}}{1-p}\left(\left(\frac{p_{N}}{q_{N}}\right)^{1-p}-1\right)
$$

Since

$$
\frac{\left(2 q_{N}\right)^{1-p}}{1-p}
$$

grows without bound as $N \rightarrow \infty$, the limit in (7) can only be finite if

$$
\lim _{N \rightarrow \infty}\left(\left(\frac{p_{N}}{q_{N}}\right)^{1-p}-1\right)=0
$$

which proves our assertion. The statement in terms of the asymptotic density $\rho$ follows immediately.

In the case of the alternating harmonic series, convergence of a rearrangement is assured if

$$
\lim _{N \rightarrow \infty} \frac{p_{N}}{q_{N}}
$$

is a finite positive number. In Corollary 3, this number must be one. This suggests that there are really at most three classes of conditionally convergent series, Namely series where a rearrangement converges to a finite limit if and only if

$$
\lim _{N \rightarrow \infty} \frac{p_{N}}{q_{N}}=1
$$

and series for which rearrangements converge if and only if this limit is a finite positive number. Finally, there is the possibility of series for which a rearrangement also converges if the limit is either 0 or if the sequence $\frac{p_{N}}{q_{N}}$ is unbounded. We will encounter aan example of such a series later in this section.

We will now extend these results to another prominent set of alternating series.

Corollary 4 Let $p<1$ and consider the series

$$
\sum_{n=2}^{\infty} \frac{1}{n(\ln n)^{p}}(-1)^{n-1}
$$


A rearrangement of this series converges to a finite limit if and only if

$$
\lim _{N \rightarrow \infty} \frac{p_{N}}{q_{N}}
$$

is finite and positive.

Proof: Observe that

$$
F(x)=\int_{2}^{x} \frac{1}{t(\ln t)^{p}} d t=\int_{\ln 2}^{\ln x} \frac{1}{s^{p}} d s .
$$

The previous Corollary implies that a rearrangement of

$$
\sum_{n=2}^{\infty} \frac{1}{n(\ln n)^{p}}(-1)^{n-1}
$$

converges to a finite limit if and only if

$$
\lim _{N \rightarrow \infty} \frac{\ln 2 p_{N}}{\ln 2 q_{N}}=1
$$

Before continuing we remark that unlike in the previous corollary we can allow $p \leq 0$ here, since the original alternating series also converges in this case and the argument of the proof of Corollary 3 still works.

We are left to show the equivalence of (9) and (8). To do this suppose first that

$$
\lim _{N \rightarrow \infty} \frac{p_{N}}{q_{N}}=L
$$

for some positive real number $L$. Next choose $\epsilon$ such that $0<\epsilon<L$. Then there exists an $N_{0}$ such that

$$
L-\epsilon<\frac{p_{N}}{q_{N}}<L+\epsilon
$$

for all $N \geq N_{0}$. Multiplication with $2 q_{N}$ yields

$$
2 q_{N}(L-\epsilon)<2 p_{N}<2 q_{N}(L+\epsilon)
$$

for all $N \geq N_{0}$. The logarithm is a strictly monotonically increasing function. Thus previous inequality implies

$$
\ln 2 q_{N}(L-\epsilon)<\ln 2 p_{N}<\ln 2 q_{N}(L+\epsilon)
$$


and

$$
\ln 2 q_{N}+\ln (L-\epsilon)<\ln 2 p_{N}<\ln 2 q_{N}+\ln (L+\epsilon) .
$$

$\ln 2 q_{N}>0$ for sufficiently large $N$, thus we may divide the inequality by this quantity to get

$$
1+\frac{\ln (L-\epsilon)}{\ln 2 q_{N}}<\frac{\ln 2 p_{N}}{\ln 2 q_{N}}<1+\frac{\ln (L+\epsilon)}{\ln 2 q_{N}}
$$

for all $N \geq N_{0}$. We take the limit as $N \rightarrow$ infty and get (9).

To show the opposite direction assume that

$$
\frac{p_{N}}{q_{N}}
$$

is unbounded. Then there exists an $N_{0}$ such that

$$
p_{N}>q_{N}
$$

for all $n \geq N_{0}$. Next observe that for every $0<\epsilon<1$ we have $x>x^{1-\epsilon}$ for all $x>1$. This implies that

$$
p_{N}>q_{N}>q_{N}^{1-\epsilon}
$$

for all $N \geq N_{0}$. Thus

$$
\frac{\ln p_{N}}{\ln q_{N}}>\frac{\ln q_{N}^{1-\epsilon}}{\ln q_{N}}=(1-\epsilon)
$$

for all $N \geq N_{0}$. Since this inequality holds for all $0<\epsilon<1$ it follows that

$$
\frac{\ln p_{N}}{\ln q_{N}} \geq 1
$$

This implies the contrapositive of $(9) \Rightarrow(8)$. If $\lim _{N \rightarrow \infty} \frac{p_{N}}{q_{N}}=0$, we apply the same $\operatorname{argument}$ to $\frac{q_{N}}{p_{N}}$. This completes the proof of the Corollary.

This last Corollary cannot be extended to the case $p=1$. In that case convergence of a rearrangement is equivalent to the condition

$$
0<\lim _{N \rightarrow \infty} \frac{\ln 2 p_{N}}{\ln 2 q_{N}}<\infty
$$


by Corollary 2. However, (10) is much weaker than (8). To see this consider the case when $p_{N}=N^{2}$ and $q_{N}=N$. Then

$$
\lim _{N \rightarrow \infty} \frac{\ln 2 p_{N}}{\ln 2 q_{N}}=\lim _{N \rightarrow \infty} \frac{\ln 2+2 \ln N}{\ln 2+\ln N}=2
$$

and

$$
\frac{p_{N}}{q_{N}}=N
$$

which is unbounded. These sequences do not satisfy $p_{N}+q_{N}=N$, but the sequences $q_{N}=[\sqrt{N}]$ and $p_{N}=N-q_{N}$ do and allow us to construct a rearrangement of the series

$$
\sum_{N=2}^{\infty} \frac{(-1)^{n}}{n \ln n}
$$

which converges to a finite number with unbounded $\frac{p_{N}}{q_{N}}$.

We finish this section by studying the series

$$
\sum_{n=2}^{\infty} \frac{1}{\ln n}(-1)^{n}
$$

A straight forward application of Theorem 1 gives that a rearrangement with a sequence of positive terms $p_{N}$ and negative terms $q_{N}$ converges to a finite number, if and only if

$$
\lim _{N \rightarrow \infty} \int_{2 q_{N}}^{2 p_{N}} \frac{1}{\ln t} d t=\lim _{N \rightarrow \infty}\left(\operatorname{Li}\left(2 p_{N}\right)-\operatorname{Li}\left(2 q_{N}\right)\right)
$$

exists and is finite. Here $\operatorname{Li}(x)$ denotes the logarithmic integral function defined as

$$
\operatorname{Li}(x)=\int_{2}^{x} \frac{1}{\ln t} d t
$$

Using l'Hospital's rule we get

$$
\lim _{x \rightarrow \infty} \frac{\operatorname{Li}(x) \ln x}{x}=1
$$

and therefore the rearrangement converges if and only if

$$
\lim _{N \rightarrow \infty}\left(\frac{2 p_{N}}{\ln 2 p_{N}}-\frac{2 q_{N}}{\ln 2 q_{N}}\right)
$$


exists and is finite. Factoring this expression as before we get

$$
\lim _{N \rightarrow \infty} \frac{2 q_{N}}{\ln 2 q_{N}}\left(\frac{p_{N} \ln 2 q_{N}}{q_{N} \ln 2 p_{N}}-1\right)
$$

which can only be finite if

$$
\lim _{N \rightarrow \infty} \frac{p_{N} \ln 2 q_{N}}{q_{N} \ln 2 p_{N}}=1
$$

From the proof of the last Corollary we have that if $\lim _{N \rightarrow \infty} \frac{p_{N}}{q_{N}}=L$ for some finite positive number $L$, then

$$
\lim _{N \rightarrow \infty} \frac{\ln 2 p_{N}}{\ln 2 q_{N}}=1
$$

It follows that in this case (11) holds if and only if $L=1$. However, (11) could hold if $\lim _{N \rightarrow \infty} \frac{p_{N}}{q_{N}}=0$ or if it diverges. To investigate this case let $r_{N}=\frac{p_{N}}{q_{N}}$, and assume that $r_{N} \rightarrow \infty$ then

$$
\frac{p_{N} \ln 2 q_{N}}{q_{N} \ln 2 p_{N}}=r_{N} \frac{\ln r_{N} 2 p_{N}}{\ln 2 p_{N}}=r_{N}\left(\frac{\ln r_{N}}{\ln 2 p_{N}}+1\right) \geq r_{N} .
$$

Clearly, this expression diverges if $r_{N} \rightarrow \infty$. On the other hand if $r_{N} \rightarrow 0$ we investigate the reciprocal of this expression in the same way. Therefore (11) can never be satisfied. We have thus shown

Corollary 5 A rearrangement of

$$
\sum_{n=2}^{\infty} \frac{1}{\ln n}
$$

converges if and only if

$$
\lim _{N \rightarrow \infty} \frac{p_{N}}{q_{N}}=1 .
$$

This last result has a slightly different interpretation in light of the prime number theorem. Let $\pi(x)$ denote the number of primes that are less than or equal to $x$, then the prime-number theorem [1, p. 74] implies that a rearrangement of this series converges if and only if

$$
\lim _{N \rightarrow \infty}\left(\pi\left(2 p_{N}\right)-\pi\left(2 q_{N}\right)\right)
$$

is finite. Or in other words if $A_{N}$ is the number of primes in the interval $\left[2 q_{N}, 2 p_{n}\right]$, then the rearrangement converges if and only if $\lim _{N \rightarrow \infty} A_{N}$ is finite. 


\section{A LIMIT COMPARISON THEOREM FOR RE- ARRANGEMENTS}

In the previous section we considered the convergence behavior of some special series. This section is devoted to a more general convergence result. Similar to the rich theory of the convergence of positive series, we will prove a comparison theorem. This will allow us to study the convergence of rearrangements of series with more complicated terms.

Theorem 2 Let

$$
\sum_{n=1}^{\infty} a_{n}(-1)^{n-1} \quad \text { and } \quad \sum_{n=1}^{\infty} b_{n}(-1)^{n-1}
$$

be two conditionally convergent series, which satisfy the assumptions spelled out in the introduction of this paper. Assume that there is a positive constant $C$ such that.

$$
\lim _{n \rightarrow \infty} \frac{a_{n}}{b_{n}}=C
$$

Then any rearrangement of

$$
\sum_{n=1}^{\infty} a_{n}(-1)^{n-1}
$$

will converge if and only if the corresponding rearrangement of

$$
\sum_{n=1}^{\infty} b_{n}(-1)^{n-1}
$$

converges.

Proof: To facilitate the proof of this theorem we need to introduce some notation. Consider a given rearrangement of the alternating series

$$
\sum_{n=1}^{\infty} a_{n}(-1)^{n-1}
$$

with associated sequence s $p_{n}$ and $q_{n}$ of positive and negative terms. Without loss of generality we assume that $p_{n}>q_{n}$ for sufficiently large $n$. Let

$$
S_{N}(a)
$$


denote the $N$-th partial sum of this rearrangement, and

$$
S_{N}(b)
$$

denote the $N$-th partial sum of the same rearrangement of

$$
\sum_{n=1}^{\infty} b_{n}(-1)^{n-1} \text {. }
$$

Moreover, let $T_{N}(a)$ and $T_{N}(b)$ denote the partial sums of the corresponding alternating series. Furthermore, let

$$
\alpha:[1, \infty) \rightarrow[0, \infty) \quad \text { and } \quad \beta:[1, \infty) \rightarrow[0, \infty)
$$

be two continuous functions with anti derivatives $A$ and $B$ such that

$$
\alpha(n)=a_{n} \quad \text { and } \quad \beta(n)=b_{n} .
$$

Finally, let

$$
B(x)=\int_{1}^{x} \beta(t) d t, \quad \text { and } \quad A(x)=\int_{1}^{x} \alpha(t) d t
$$

Let $\epsilon>0$ then there exists an $N_{0}$ such that

$$
b_{n}(C-\epsilon)<a_{n}<b_{n}(C+\epsilon)
$$

for all $n \geq \frac{q_{N}}{2}+1$. Let $N \geq N_{0}$, then

$$
\begin{aligned}
S_{N}(a) & =T_{q_{N}}(a)+\sum_{n=q_{N}+1}^{p_{N}} a_{2 n-1} \\
& \leq T_{q_{N}}(a)+(C+\epsilon) \sum_{n=q_{N}+1}^{p_{N}} b_{2 n-1}
\end{aligned}
$$

Analogously, we get

$$
\begin{aligned}
S_{M}(a) & =T_{q_{M}}(a)+\sum_{n=q_{M}+1}^{p_{M}} a_{2 n-1} \\
& \geq T_{q_{M}}(a)+(C-\epsilon) \sum_{n=q_{M}+1}^{p_{M}} b_{2 n-1}
\end{aligned}
$$


for $M \geq N_{0}$. Subtracting the second inequality from the first we get

$$
\begin{aligned}
S_{N}(a)-S_{M}(a) \leq & T_{q_{N}}(a)-T_{q_{M}}(a)+C \sum_{n=q_{N}+1}^{p_{N}} b_{2 n-1}-C \sum_{n=q_{M}+1}^{p_{M}} b_{2 n-1} \\
& \quad+\epsilon \sum_{n=q_{N}+1}^{p_{N}} b_{2 n-1}+\epsilon \sum_{n=q_{M}+1}^{p_{M}} b_{2 n-1} \\
\leq & T_{q_{N}}(a)-T_{q_{M}}(a)+C\left(T_{q_{M}}(b)-T_{q_{N}}(b)\right) \\
& +C\left(S_{N}(b)-S_{M}(b)\right)+\epsilon \sum_{n=q_{N}+1}^{p_{N}} b_{2 n-1}+\epsilon \sum_{n=q_{M}+1}^{p_{M}} b_{2 n-1} \\
\leq & \left|T_{q_{N}}(a)-T_{q_{M}}(a)\right|+C\left|T_{q_{M}}(b)-T_{q_{N}}(b)\right| \quad S_{M}(b) \mid+\epsilon \sum_{n=q_{N}+1}^{p_{N}} b_{2 n-1}+\epsilon \sum_{n=q_{M}+1}^{p_{M}} b_{2 n-1}
\end{aligned}
$$

In this step we used the decomposition of $S_{N}(b)$ into $T_{q_{M}}(b)$ and a positive remainder term, and the fact that $x \leq|x|$. On the right hand side of the last inequality we may interchange $M$ and $N$ without changing the value of the right hand side. This implies that the same inequality applies to

$$
S_{M}(a)-S_{N}(a)
$$

and therefore

$$
\begin{aligned}
\left|S_{N}(a)-S_{M}(a)\right| \leq \mid & \left|T_{q_{N}}(a)-T_{q_{M}}(a)\right|+C\left|T_{q_{M}}(b)-T_{q_{N}}(b)\right| \\
& +C\left|S_{N}(b)-S_{M}(b)\right|+\epsilon \sum_{n=q_{N}+1}^{p_{N}} b_{2 n-1}+\epsilon \sum_{n=q_{M}+1}^{p_{M}} b_{2 n-1}
\end{aligned}
$$

Next, from the proof of Theorem 1 we have that

$$
\sum_{n=q_{N}+1}^{p_{N}} b_{2 n-1} \leq \frac{1}{2} \int_{2 q_{N}}^{2 p_{N}} \beta(t) d t+b_{2 q_{N}+1}=B\left(2 p_{N}\right)-B\left(2 q_{N}\right)+b_{2 q_{N}+1} .
$$

Now assume that $S_{N}(b)$ converges, then by Theorem $1 B\left(2 p_{N}\right)-B\left(2 q_{N}\right)$ converges to a finite number and hence there exists a $K>0$ such that

$$
B\left(2 p_{N}\right)-B\left(2 q_{N}\right)+b_{2 q_{N}+1}<K
$$


for all $N$. Next, since $S_{N}(b), T_{N}(a)$, and $T_{N}(b)$ all converge, they are Cauchy sequences and there exists a $M_{0}$ such that

$$
\begin{array}{r}
\left|T_{q_{N}}(a)-T_{q_{M}}(a)\right|<\epsilon \\
\left|T_{q_{N}}(b)-T_{q_{M}}(b)\right|<\epsilon \\
\left|S_{N}(b)-S_{M}(b)\right|<\epsilon
\end{array}
$$

Hence, for $M, N \geq \max \left\{N_{0}, M_{0}\right\}$

$$
\left|S_{N}(a)-S_{M}(a)\right|<\epsilon+2 C \epsilon+2 K \epsilon
$$

and therefore it is a Cauchy sequence and it converges. The opposite direction is proved completely analogously.

We illuminate the use of Theorem 2 by an example. Consider the series

$$
\sum_{n=1}^{\infty} \frac{(-1)^{n}}{\ln \sin \frac{1}{n}}
$$

The reader can easily verify that

$$
a_{n}=\frac{-1}{\ln \sin \frac{1}{n}}
$$

satisfies the conditions of both theorems. Now using l'Hospital's rule we get

$$
\lim _{x \rightarrow \infty} \frac{-\ln \sin \frac{1}{x}}{\ln x}=1
$$

Therefore, by Corollary 6, any rearrangement of this series converges if and only if

$$
\lim _{n \rightarrow \infty} \frac{p_{n}}{q_{n}}=1
$$

\section{Remarks:}

1. To best of our knowledge, Theorem 2 is, at least in the form given, a new result, albeit not very useful. A. Pringsheim [7] does a comparison of conditionally convergent series with the alternating harmonic series, by comparing whether

$$
\lim _{n \rightarrow \infty} n a_{n}
$$


is finite or not. This result follows from Theorem 2 by using the alternating harmonic series as one of the two series used in Theorem 2.

2. The condition that

$$
0<\lim _{n \rightarrow \infty} \frac{a_{n}}{b_{n}}<\infty
$$

is only a sufficient condition for rearrangements of the two series two converge together. As we have seen in Corollaries 2 and 3 rearrangements of,

$$
\sum_{n=1}^{\infty} \frac{(-1)^{n-1}}{n} \text { and } \sum_{n=2}^{\infty} \frac{\log n}{n}(-1)^{n}
$$

converge together, but the condition of Theorem 2 is obviously not satisfied.

3. The entire paper only applies to series with eventually decreasing terms. There are, however series which do not satisfy this property and which are still conditionally convergent and subject to Riemann's Theorem.

\section{References}

[1] Apostol, Tom M., Introduction to Analytic Number Theory, Springer Verlag, New York, 1976.

[2] R. Beigel, Rearranging Terms in Alternating Series, Mathematics Magazine, Vol. 54, No. 5 (1981), 244-246.

[3] F. Brown, L. O. Cannon,J. Elich,D. Wright, On Rearrangements of the Alternating Harmonic Series, The College Mathematics Journal, Vol. 16, No. 2 (1985), 135-138.

[4] R. Courant, F. John, Introduction to Calculus and Analysis, Springer Verlag, New York, 1989.

[5] C. C. Cowen, K. R. Davidson, R. P. Kaufman, Rearranging the Alternating Harmonic Series, A Century of Calculus, Part II, (edited by T. Apostol, D. Mugler, D. Scott, A. Sterrett, and A. Watkins), MAA, Washington DC, 1992) 
[6] K. Knopp, Theory and Application of Infinite Series, Second English Edition, Hafner Publishing, New York, 1971.

[7] A. Pringsheim, Ueber die Werthveränderungen bedingt convergenter Reihen und Producte, Mathematische Annalen, XXII (1883), 455-503.

[8] P. Schaefer, Sums of Rearranged Series, The College Mathematics Journal, Vol. 17, No. 1 (1986), 66-70. 